\title{
TECNOLOGÍAS MODULARES DE CLIMATIZACIÓN SOLAR PASIVA PARA EL HÁBITAT RESIDENCIAL Y EL DE LA PRODUCCIÓN
}

Eje 2: Tecnología para la construcción sustentable

\author{
Jodra Juan Ignacio' \\ Viegas Graciela Melisa² \\ San Juan Gustavo Alberto 3 \\ Discoli Carlos Alberto 4
}

1,2,3,4 Instituto de Investigaciones y Políticas del Ambiente Construido (IIPAC) Facultad de Arquitectura y Urbanismo (FAU), Universidad Nacional de La Plata (UNLP). Argentina. 1juanjodra@gmail.com 2gachiviegas@yahoo.com.ar; ${ }^{3}$ gustavosanjuan60@hotmail.com; ${ }^{4}$ discoli@rocketmail.com

\section{RESUMEN}

El trabajo expone el desarrollo de un sistema acumulador / amortiguador de calor solar térmico de diseño modular y tecnología industrializable. Se trata de un sistema de funcionamiento pasivo y aplicación versátil, orientado a satisfacer requerimientos de climatización y/o estabilización térmica tanto de espacios residenciales como de espacios productivos del sector agropecuario. Se muestra el estudio de antecedentes, el proceso de diseño y los prototipos desarrollados teniendo en cuenta componentes constructivos, geometrías y materialidades. Se presentan los resultados de ensayos de operación del sistema, instalado sobre dos tipos de espacios. En un primer ensayo, se demostró un comportamiento térmico favorable en un espacio productivo tipo invernadero, donde se registró una reducción de la amplitud térmica en su interior, en comparación a la situación del invernadero sin el sistema. Así mismo, se disminuyeron los picos de calentamiento diurno y se estabilizaron las temperaturas de base durante el período nocturno, evitando el fenómeno de inversión térmica. En un segundo ensayo, se instaló el sistema configurado como muro acumulador de calor en un box de ensayos de $9 \mathrm{~m} 2$, equiparable a un dormitorio. Se obtuvieron valores de rendimiento de carga superiores al $60 \%$ y tiempos de retardo en la onda térmica para calefacción, entre 4 y 4,3 horas. De esta forma el sistema demostró su aptitud para proporcionar un paquete de energía de base para la climatización de este tipo de espacios.

\section{PALABRAS CLAVES: ENERGÍAS RENOVABLES - SISTEMAS SOLARES PASIVOS - INVERNADEROS - VIVIENDAS}




\section{INTRODUCCIÓN}

La energía en la Argentina si bien es una dimensión estratégica, hoy día representa un problema estructural, tanto desde el plano ambiental ${ }^{1}$, como desde sus problemas de abastecimiento, merma de producción, costos y tarifas ${ }^{2 ;}$ configurando así un estado de crisis institucional. La limitada disponibilidad de recurso energético ${ }^{3}$, fundamentalmente de origen fósil, combinada con la ajustada capacidad de las redes de transporte de energía, reafirman la necesidad de atacar esta problemática de manera simultánea desde múltiples aristas. Ante esto, y desde un enfoque energético-ambiental, las acciones a tomar deben contemplar no sólo la inversión en infraestructura de generación y transporte, sino también en la incorporación de medidas que promuevan la eficiencia energética y la incorporación de fuentes renovables a la matriz energética.

De esta forma, ante el panorama energético-ambiental actual, se refuerza el interés sobre todas aquellas tecnologías que posibiliten el ahorro energético y el aprovechamiento de fuentes renovables de energía. En particular aquellas orientadas a la construcción sustentable, y que incorporen en sus criterios aplicaciones solares pasivas a partir de los requerimientos locales, ya que pueden presentar una opción valiosa para afrontar estas problemáticas.

Se denominan tecnologías solares pasivas, a aquellas que permiten el aprovechamiento y utilización de la energía de la radiación solar sin necesidad de consumir energía desde otras fuentes ni de la operación o realización de maniobras por parte del usuario. En otras palabras, son sistemas tecnológicos que funcionan de manera autónoma permitiendo el aprovechamiento del recurso solar para distintas finalidades. En este trabajo, se aborda una aplicación de tipo térmico, presentándose el desarrollo de un sistema de climatización y estabilización orientado a espacios residenciales y agro-productivos.

\subsection{Antecedentes}

El origen de los sistemas pasivos másicos tradicionales se remonta a finales del siglo XIX, cuando Edward Morse (1881) patenta su trabajo: "Warming and ventilating apartements by thesun's rays". Sin embargo, su difusión, implementación y desarrollo comenzaron recién unos 80 años más tarde, en la década del sesenta, con las construcciones realizadas por el Ing. Felix Trombe y el Arq. Jacques Michel en las casas pasivas de la localidad francesa de Font-Romeu-Odeilo-Via, en los Pirineos orientales. Esta intervención dinamizó la temática solar térmica extendiendo el conocimiento de estas tecnologías bajo el nombre de "muros Trombe-Michel", y dejó en segundo plano los orígenes mencionados. Posteriormente y hasta la actualidad, se iniciaron múltiples desarrollos explorando diferentes variantes sobre la materialidad de la masa de acumulación de calor (A. Di Vecchia, et al, 1979) y la aplicación en diferentes localizaciones (distintos climas y recurso solar) llegándose a establecer criterios básicos para su implementación en edificios e incorporando pautas aplicables a la construcción y utilización sistemática (Balcomb, et al 1981).

Contemporáneamente en Argentina también se realizaron significativos desarrollos solares pasivos, mayoritariamente aplicados al sector residencial en varios centros de investigación emplazados en diferentes regiones y climas del país (Alanis, 1978); (Guerrero et al., 1979; Discoli

\footnotetext{
${ }^{1}$ Según el IPCC, la emisión de gases de efecto invernadero (GEI) asociada al consumo de combustibles fósiles presenta una relación causal directa con diversos efectos que atentan sobre el equilibrio medioambiental del planeta. (IPCC, 2014).

${ }^{2}$ Relacionada con las falencias de infraestructura como con la desregulación de las tarifas (Chévez, 2016)

${ }^{3}$ El $11 \%$ de la demanda de gas natural se abastece por medio de importación. Fuente BEN, 2016.
} 
et al., 1985). En las últimas cuatro décadas, se realizaron aplicaciones en climas fríos y secos, con recurso solar alto y gran amplitud térmica; requiriendo para estos casos sistemas másicos con acumulación preferentemente sólida (Piedra, Ladrillo, Hormigón $-\mathrm{H}^{\circ} \mathrm{A}^{\circ}$, etc.), desfasando la carga entre el período diurno y el nocturno, y reiniciando la carga en el período diurno próximo mediato (Lesino et al., 1981), (Discoli et al, 1986). Recientemente se continuaron desarrollando sistemas para zonas climáticas templadas mediterráneas (Prov. de Buenos Aires) (ver Figura 2) mediante el desarrollo de tecnologías que cuentan con características de agilidad en el proceso de carga de calor, mayor capacidad de acumulación, inercia térmica, niveles de desfasaje intermedios (4 a 6 hs.), y descarga de calor semi-controlada (Patente Discoli \& San Juan, 2010) (Discoli, Viegas \& San Juan, 2012).

Con respecto a los espacios destinados a la agro-producción, el uso de sistemas solares térmicos pasivos se circunscribe en general a la implementación de invernáculos livianos tradicionales. En algunos casos, se cuenta con sistemas auxiliares de climatización para estabilizar el ambiente durante los períodos adversos en el ciclo diurno/nocturno, utilizándose principalmente equipos convencionales (combustibles sólidos, líquidos y gaseosos) y biomasa, en la mayoría de los casos con ignición directa. Los mismos permiten mantener una temperatura establecida en los espacios, y reducir la amplitud térmica. Se busca así, evitar diversos daños en los cultivos producidos por picos de calentamiento indeseado durante el período diurno, o por heladas durante la fase nocturna, aunque con significativos gastos operativos en combustible e importantes niveles de contaminación directa e indirecta. Si bien estos sistemas son los más difundidos, existen diferentes antecedentes que plantean la utilización de energías renovables acumulando energía térmica en reservorios auxiliares, combinadas con sistemas activos de bombeo o ventiladores, para la circulación de un caudal de agua (Suárez, 2012), o aire (Bistoni, Iriarte, \& Saravia, 2004; Juanicó, Piacentini, \& La Mattina, 2011), como fluidos de intercambio (ver Figura1). En su mayoría utilizan reservorios térmicos auxiliares con ganancia solar directa externos al espacio de producción.

En cuanto a los sistemas pasivos, su principio básico consiste en la utilización de la irradiación solar directa (ganancia solar directa), combinada con elementos de acumulación térmica (masa) con elevada capacidad calorífica, distribuidos dentro del propio espacio de producción (criaderos, parideras, invernaderos, etc.). De esta manera se logra amortiguar (bajo el concepto de inercia térmica) los picos de temperatura que se producirían con el sobrecalentamiento diurno, a la vez que se incrementa la temperatura de la masa de acumulación distribuida. Dicha energía es entregada durante el periodo nocturno aportando a mantener el espacio en un mayor nivel térmico. Un buen ejemplo de ello son los invernaderos solares pasivos tradicionales chinos (LiHong et al., 2010), desarrollados durante la década de 1980. Otros antecedentes más recientes, plantean soluciones no industrializables utilizando materiales reciclados como botellas PET para la conformación de muros inerciadores con acumulación en agua (Esteves \& Gelardi, 1999).

En función de los antecedentes estudiados, este trabajo aborda dicha problemática, planteando una solución tecnológica solar pasiva. Se diseña un sistema modular industrializable que utiliza los principios de acumulación de calor y amortiguamiento térmico permitiendo una utilidad versátil, tanto el acondicionamiento y la estabilización de espacios agro-productivos, así como para la climatización de viviendas y otros espacios edilicios. 


\section{DESARROLLO}

En los puntos que siguen, se presentan las etapas de diseño, desarrollo, fabricación y ensayos de un sistema de alta capacidad de carga térmica y de uso versátil, denominado "Sistema Acumulador/Amortiguador Térmico Solar Pasivo". La metodología se detalla en las siguientes etapas:

i. Evaluación técnica de antecedentes.

ii. Diseño de los prototipos.

iii. Ensayo de muro acumulador de calor.

iv. Ensayo de amortiguador térmico en invernadero.

\subsection{Evaluación técnica de antecedentes}

Previo al diseño de los prototipos, se trabajó en la búsqueda y análisis de antecedentes, seleccionando a partir de la misma los sistemas tecnológicos más significativos. Estos se compararon mediante una matriz de evaluación de acuerdo a Jodra et al (2017), cuyo resultado permitió adoptar una tecnología de referencia sobre la cual se basó el concepto y diseño de los prototipos desarrollados. Se trata del sistema patentado por Discoli \& San Juan (2010) que incorpora la utilización de vainas plásticas con agua encriptada insertas en bloques de hormigón industrializados (Ver Figura 1). Posteriormente, se orientó el trabajo de diseño en base a este antecedente, manteniendo la materialidad, pero mejorando los atributos de armado/montaje modificando la modularidad (menor peso por pieza manipulada); versatilidad de aplicación, y diversidad de configuraciones.
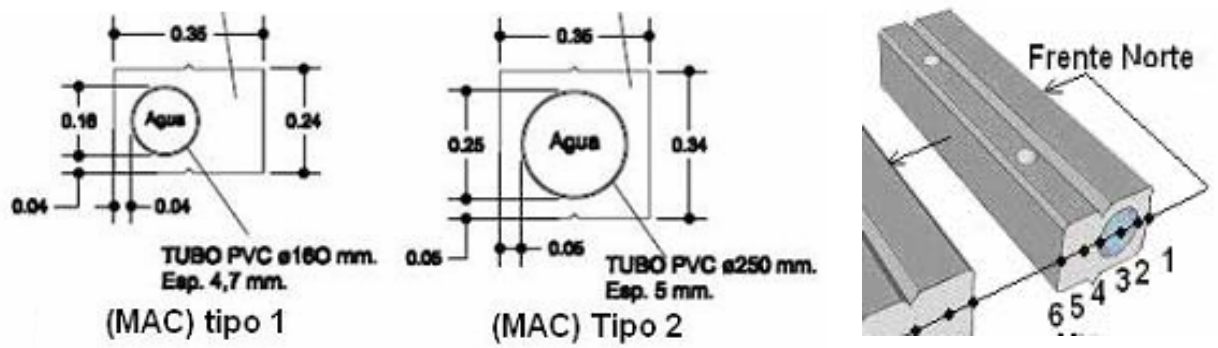

Fig. 1: Prototipo de MAC mixtos. Discoli \& San Juan, 2010.

\subsection{Diseño conceptual y técnico de los prototipos}

Se trabajó sobre el diseño conceptual y técnico de distintos prototipos, utilizando herramientas de apoyo como Excel, AutoCAD y Sketch Up. El diseño contempló el desarrollo de dos tipos de piezas en función de la materialidad mixta planteada (hormigón + agua). Por un lado piezas sólidas premoldeadas de hormigón pigmentado (se incorporó negro de humo en un $2 \%$ ), y por otro, para contener el agua se diseñaron vainas plásticas estancas conformadas a partir de tubos comerciales de PVC de $160 \mathrm{~mm}$ de diámetro y sus correspondientes tapas ciegas. Las mismas pueden combinarse permitiendo la conformación de muros acumuladores de calor (MAC) o también diversas morfologías de masas de acumulación térmica. En cuanto a sus aplicaciones, su diseño permite implementar en su armado diferentes configuraciones orientadas tanto a espacios residenciales como al sector agro-productivo. El sistema podría en tanto, formar parte de una 
vivienda, constituyendo un sistema pasivo de climatización tipo MAC incorporado a la fachada o a la cara norte de la misma; o bien podría incorporarse dentro de la misma, para su empleo como acumulador térmico en función de un planteo de ganancia solar directa. Así mismo, en espacios agro-productivos como invernáculos, parideras y criaderos, entre otros, puede instalarse como masa de acumulación térmica. Sus diferentes configuraciones dependerán de los requerimientos de operación del espacio de producción, y del recurso helio-energético disponible, entre otros factores (ver Figura 2).
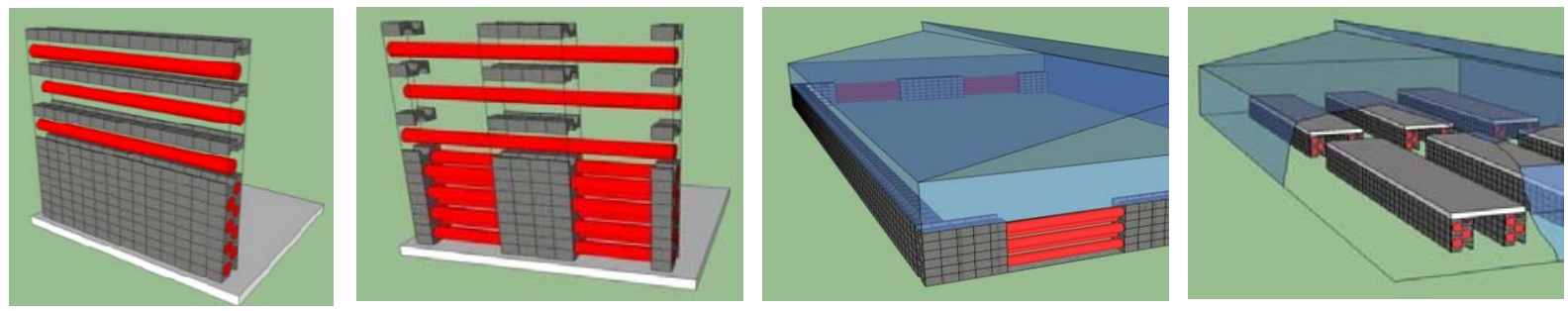

Fig. 2: Diseño conceptual. Configuraciones y aplicaciones del sistema

Para el diseño de las piezas sólidas, se optó por desarrollar dos diferentes prototipos variando fundamentalmente la geometría y el volumen de líquido relativo según la disposición de los tubos. Se estudiaron diferentes variantes optimizando y realimentando el proceso con consultas a fabricantes de pre-moldeados a los efectos de lograr su viabilidad tecnológica en cuanto a su nivel de terminación, ajustes dimensionales en el ensamble y costos. Completada la etapa de diseño se encomendó a una empresa de pre-moldeados de hormigón (BLOCKY SRL), la fabricación de los moldes y de la cantidad de bloques necesarios. El diseño y construcción de los sistemas se llevaron a cabo en el marco de los proyectos PITAP-UNLP ${ }^{4}$, y PIP 097 financiado por CONICET 5 . La Tabla 1 muestra cuantitativamente las características termo-físicas del sistema en sus dos versiones con diferente relación Hormigón + Agua.

\begin{tabular}{|l|c|c|}
\cline { 2 - 3 } \multicolumn{1}{c|}{} & Tubo Simple & Dosbolillo \\
\hline Diámetro de tubos $(\mathrm{mm})$ & 160 & 160 \\
\hline Profundidad (espesor de muro) $(\mathrm{mm})$ & 350 & 350 \\
\hline Altura $(\mathrm{mm})$ & 200 & 140 \\
\hline Ancho de pieza $(\mathrm{mm})$ & 200 & 200 \\
\hline$\%$ V $/ \mathrm{N}$ de $\mathrm{H}_{2} \mathrm{O}$ & $29 \%$ & $41 \%$ \\
\hline Peso estimado $\left(\mathrm{kg} / \mathrm{m}^{2}\right)$ & 649.36 & 597.66 \\
\hline Densidad estimada $\left(\mathrm{kg} / \mathrm{m}^{3}\right)$ & 1855.31 & 1707.60 \\
\hline Volumen de agua $\left(\mathrm{l} / \mathrm{m}^{2}\right)$ & 100.53 & 143.62 \\
\hline Volumen de agua $\left(\mathrm{l} / \mathrm{m}^{3}\right)$ & 287.23 & 410.34 \\
\hline Peso por pieza (teórico) $(\mathrm{kg})$ & 21.95 & 12.71 \\
\hline Piezas $/ \mathrm{m}^{2}$ de muro & 25.0 & 35.7 \\
\hline Capacidad Calorífica/m $/ \mathrm{m}^{2}$ de muro $\left(\mathrm{kJ} / \mathrm{m}^{2} \mathrm{~K}\right)$ & 925.75 & 1018.90 \\
\hline Capacidad Calorífica volumétrica $\left(\mathrm{kJ} / \mathrm{m}^{3} \mathrm{~K}\right)$ & 2644.99 & 2911.13 \\
\hline
\end{tabular}

Tabla 1: Especificaciones del sistema en sus dos versiones

\footnotetext{
4“Producción social del hábitat sustentable (PSHs)". PITAP-UNLP. Director: Dr. Gustavo San Juan. 2015/2017.

5 "Desarrollo de tecnologías y pautas de reciclado masivo de la envolvente edilicia residencial orientado al uso racional y eficiente de la energía en áreas urbanas"2014/2016 (Dirección: Dr. Carlos Discoli / Dra. Irene Martini).
} 


\subsection{Ensayo de Muro Acumulador de Calor en un BOX tipo ‘dormitorio’}

A fin de evaluar el desempeño del sistema en su aplicación como muro acumulador de calor, se realizaron ensayos en laboratorio a cielo abierto, instalando distintas configuraciones de MAC en la fachada norte de un módulo de ensayos de $9 \mathrm{~m} 2$ (escala 1:1). Mediante adquisidores de datos (Novus Field Logger y $\mathrm{HOBO}$ ) se registraron valores de temperaturas (ver Gráfico 1), radiación en el plano vertical, y condiciones ambientales (Estación meteorológica Davis Vantage Pro2). A continuación se muestran los resultados del ensayo realizado para un muro de descarga directa en el que se utilizó el diseño de Tubo Simple (ver Tabla 1). El mismo quedó conformado por 81 bloques y 9 vainas de agua estancas con una masa total de $2308 \mathrm{~kg}$.

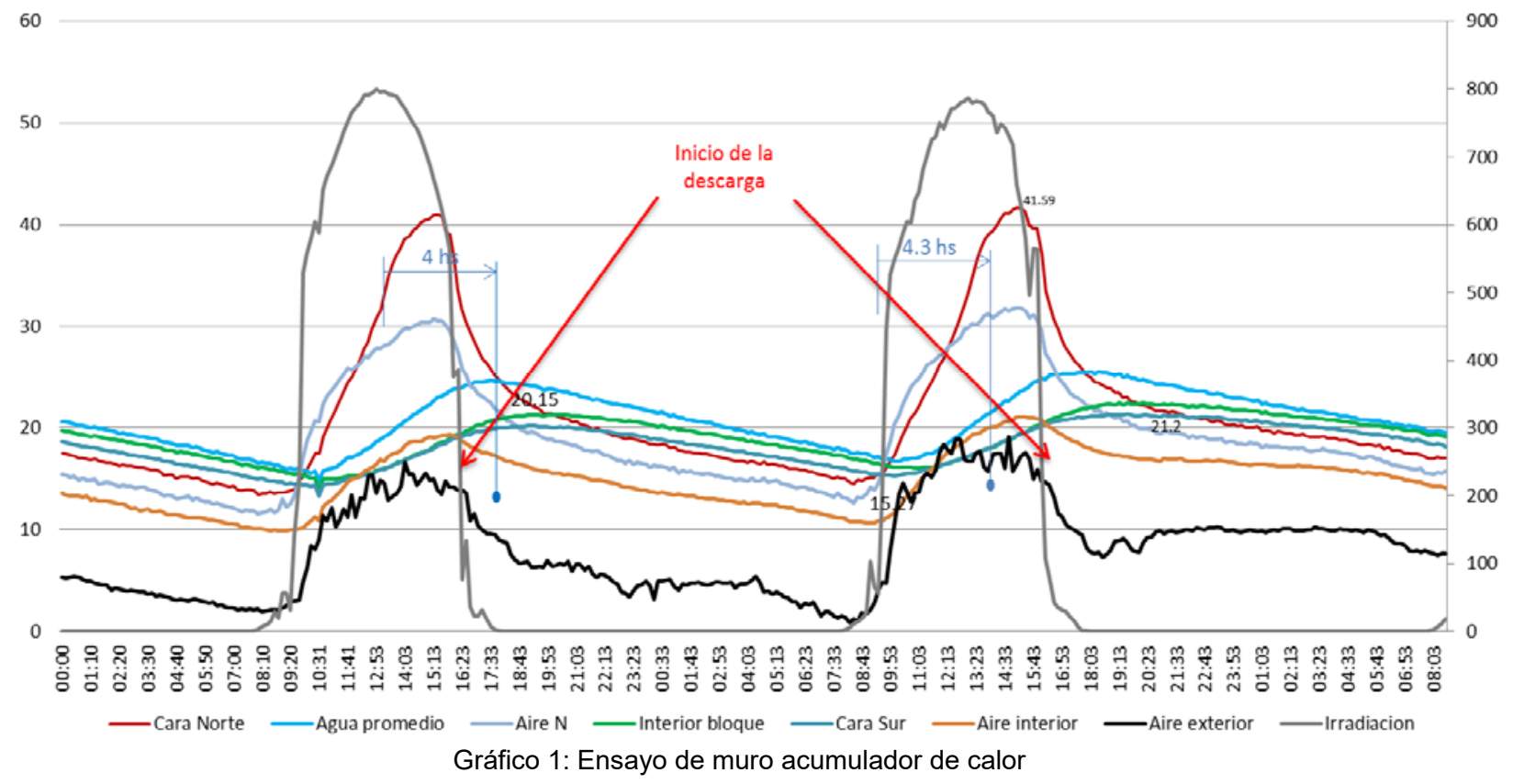

Se seleccionaron para la comparación, los días de mayor nivel de irradiación en el período (cielo despejado). De acuerdo al Gráfico 1, el retardo de la onda térmica se registró entre 4 y 4,3 hs. En tanto el ciclo de descarga del muro comienza alrededor de las 16 hs, aproximadamente una hora después de la finalización del ciclo de carga.

El amortiguamiento de la onda térmica fue de $20^{\circ} \mathrm{C}$, de los cuales $16^{\circ} \mathrm{C}$ corresponden al amortiguamiento en la transferencia conductiva desde el hormigón (lado $\mathrm{N}$ ) hacia el agua en el interior de la vaina plástica. En relación a esto, se observa una diferencia o salto entre la temperatura alcanzada por la cara norte y el resto de las temperaturas medidas en la sección del muro. Dicho comportamiento se puede relacionar con una deficiencia en el contacto térmico entre bloques y tubos. Aun así, al contabilizar la energía térmica acumulada durante la fase de carga se obtuvo un rendimiento térmico de $58 \%$ y $61 \%$ para el primer y segundo día, respectivamente.

A su vez, las curvas permiten apreciar la entrega de calor durante el ciclo de descarga, observándose al final del mismo, que el muro aun cuenta en su cara interior con una temperatura entre 4 y $5^{\circ} \mathrm{C}$, superior a la del aire interior. Por otra parte, sobre las últimas dos horas de dicho ciclo, se equiparan las temperaturas medidas en el agua y en el interior del bloque evidenciando la dinámica interna de transferencia de calor. Finalmente, en cuanto a la condición climática, se 
observa que la temperatura mínima registrada en la madrugada del segundo día de ensayo $\left(0.82^{\circ} \mathrm{C}\right)$ corresponde al tercer día más frío del período invernal.

\subsection{Ensayo de Amortiguador térmico en un BOX tipo 'Invernadero'}

El siguiente ensayo se realiza a fin de evaluar el desempeño y las potencialidades del sistema para estabilizar térmicamente espacios de producción. A tal fin se plantea el desarrollo del mismo en laboratorio a cielo abierto (LCA) utilizando un espacio similar a un invernadero de cultivo pequeño de $9 \mathrm{~m} 2$ conformado a partir por una cubierta de polietileno de $200 \mu \mathrm{m}$ tensada sobre una estructura metálica liviana. Se plantea un análisis comparativo entre una situación base 'sin sistema', en la que se registran las fluctuaciones térmicas sin ningún dispositivo de climatización; y luego con la incorporación del sistema conformando un amortiguador térmico con una masa total de 864 kg (Ver Figura 4).
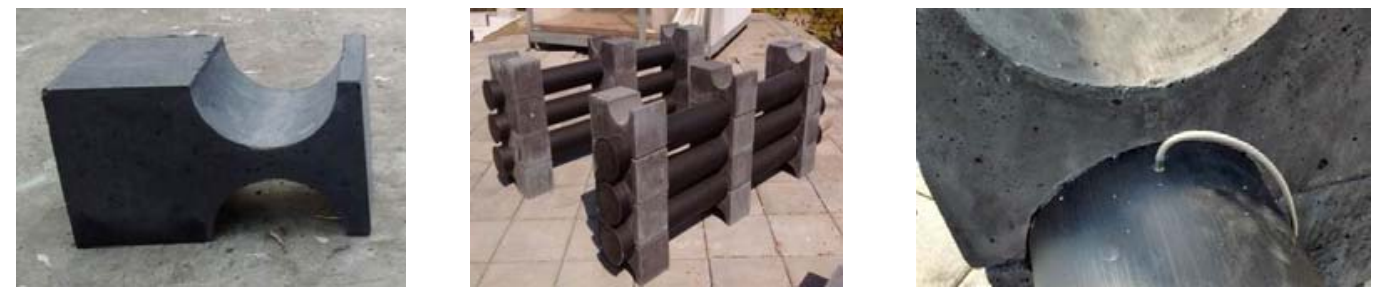

Fig. 4: Piezas de "tubo simple", vista del sistema armado y detalle de la instrumentación.

Esta etapa permite realizar un análisis, comparado con el caso anterior, verificando las potencialidades y aptitudes térmicas de dicho sistema. La instrumentación se resolvió mediante adquisidores de datos $\mathrm{HOBO}$, registrándose en total 9 parámetros de temperaturas de aire y diferentes puntos del sistema. Los datos climáticos y de radiación horizontal se obtuvieron a través de una central meteorológica Davis Vantage PRO 2.

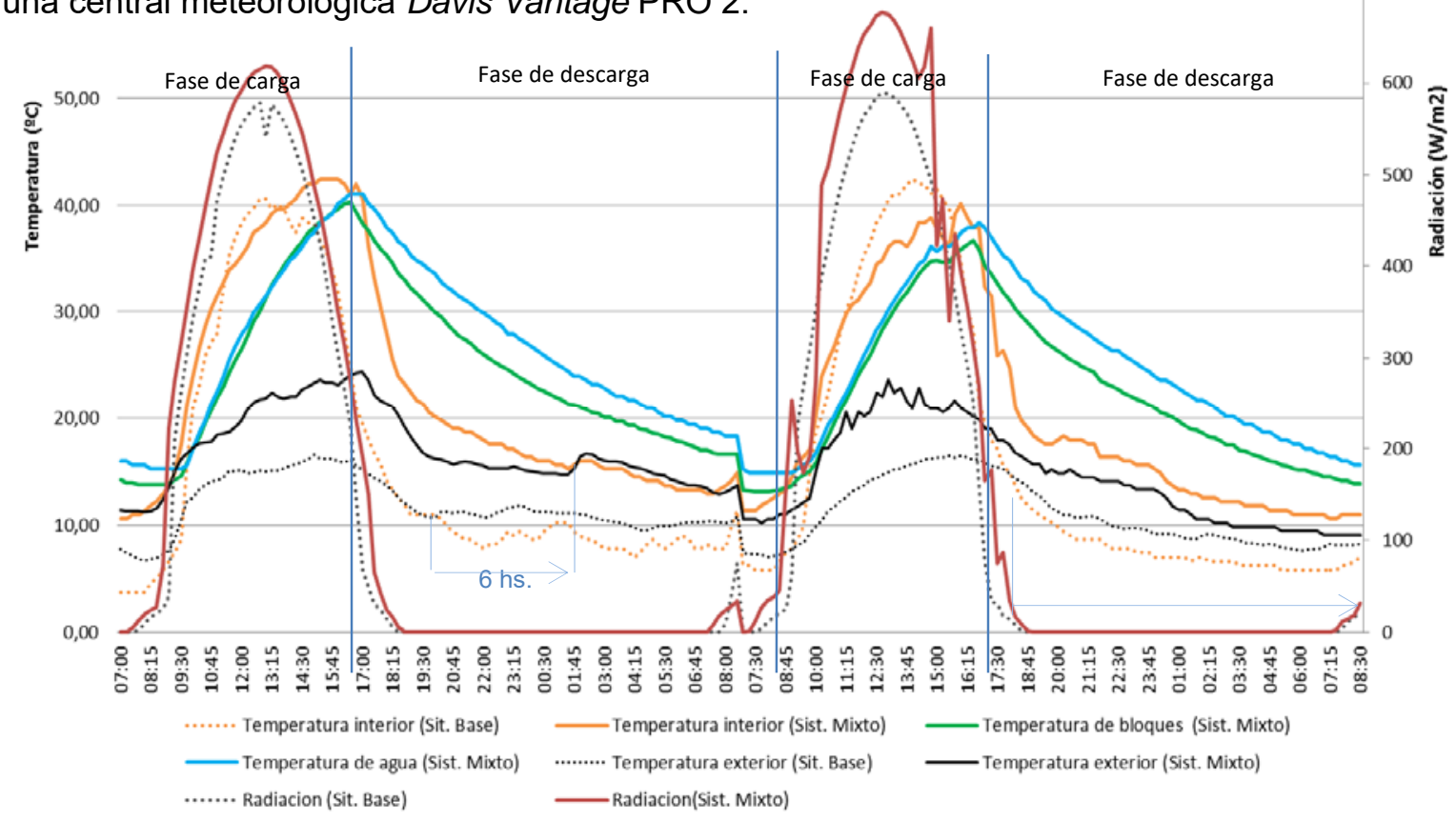

Gráfico 2: Situación Base vs. Sistema amortiguador térmico

El Gráfico 2 muestra los estados térmicos y la irradiación del invernadero vacío y del invernadero cargado con el sistema mixto. A partir del mismo, es posible analizar el comportamiento de carga- 
descarga del sistema, observándose que la fase de carga comienza dos horas después del amanecer, en el momento en que el invernadero comienza a recibir irradiación solar directa. Dicho proceso se sostiene mientras continua esta condición, hasta las 16:30 hs. Luego comienza la fase de descarga en la que ocurre el aporte térmico del sistema hasta el comienzo de un nuevo ciclo.

Durante la fase de carga en el invernadero con amortiguador térmico, se advierte una disminución en la pendiente de la curva de temperatura de aire interior, mientras que el valor de pico se registró con un retardo de unas dos horas respecto a la situación Base. La carga del sistema continua durante algunos minutos más, incorporando calor aún en el comienzo del enfriamiento interior (intersección entre las curvas de temperatura interior y temperaturas del sistema). En la fase de descarga, la temperatura del invernadero evoluciona en ambos casos con una mayor pendiente (decreciente) en su comienzo, pasando luego a una etapa de "valle", con menor variación durante el período nocturno. En las curvas de temperatura del sistema (bloques y agua) se aprecia una dinámica estable con leves variaciones en su pendiente, permitiendo sostener el aporte térmico durante todo el período nocturno.

El aporte térmico total, correspondiente a los bloques sólidos y las vainas con agua encriptada, fue de 36.52 y $35.92 \mathrm{MJ}$ en el primer y segundo día respectivamente. Por otra parte, se observa que luego del ocaso, en la situación base, la temperatura interior desciende por debajo de la exterior manteniéndose esta relación durante toda la fase nocturna. Este fenómeno de "inversión térmica" se encuentra asociado a las características del polietileno utilizado (tipo LD sin filtro para la radiación infrarroja), y a la consiguiente emisión radiativa hacia la atmósfera durante el período nocturno. Con la incorporación del sistema, se advierte su aparición en el primer ciclo comparado, con un retardo de aproximadamente 6 hs. respecto a la situación base; mientras que en el segundo ciclo, no se registró dicho fenómeno. Se observa además, que su aparición en el primer ciclo está fuertemente determinada por un incremento repentino de la temperatura exterior que se registró alrededor de las 2:00 a.m. junto con la llegada de ráfagas de viento norte (viento cálido).

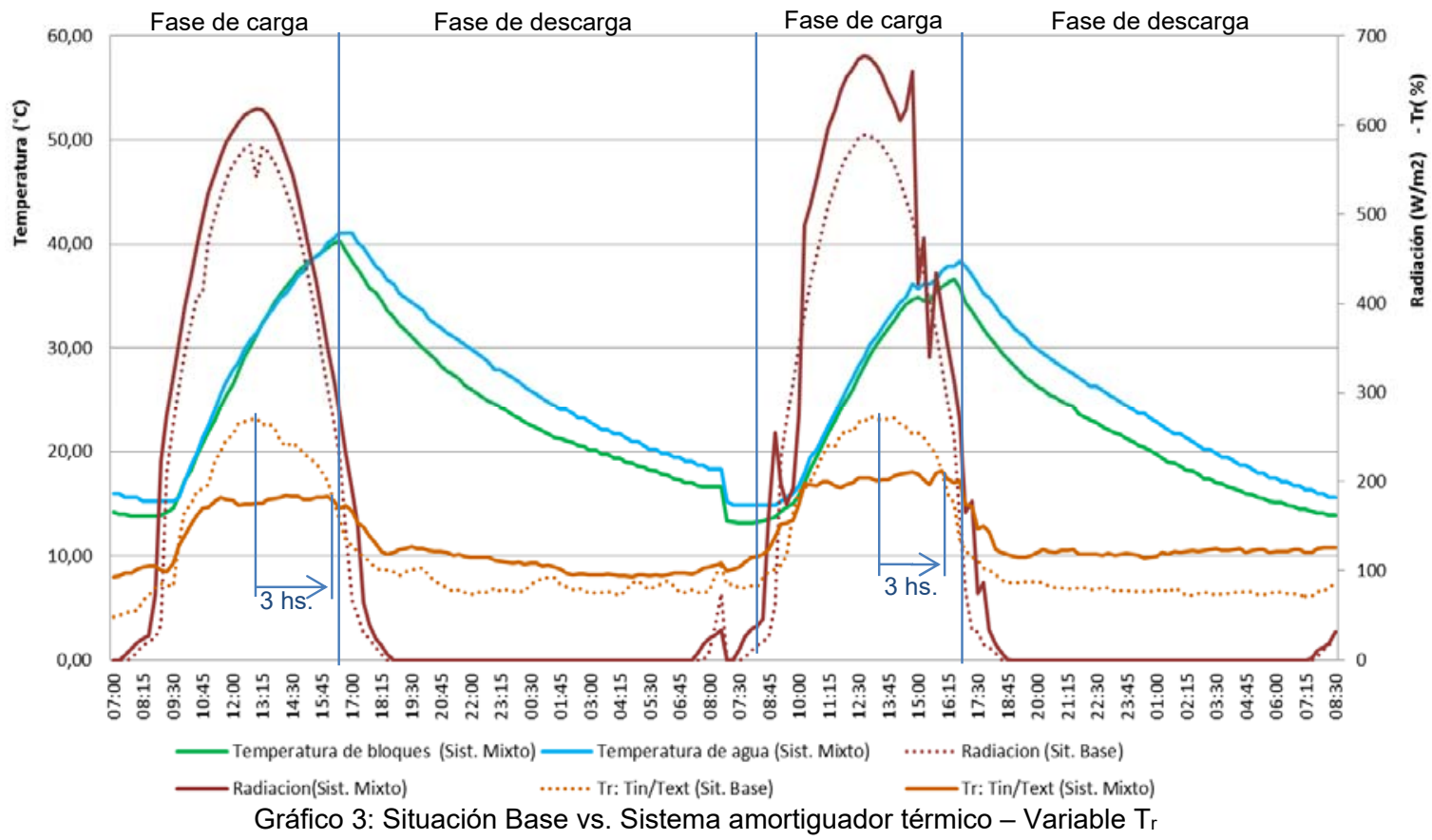


Finalmente, a los efectos de compatibilizar y poder normalizar el análisis comparado, dado que los días de ensayos no reproducen exactamente los mismos parámetros climáticos (temperatura exterior e irradiancia) entre una medición y la subsiguiente, se trabajó también con una variable indirecta de temperatura relativa definida como:

$$
T_{p}(\%)=T_{m e} / T_{w E}=100 \% \text { dónde: } \begin{aligned}
& T_{\text {int }} \text { corresponde a la temperatura del aire interior del recinto } \\
& T_{\text {ext }} \text { a la temperatura ambiente exterior. }
\end{aligned}
$$

A partir del trabajo con dicha variable, analizando la fase de carga, se observan en la variable Tr, (ver Gráfico 3) un retardo medio de tres horas y una disminución o amortiguamiento de entre el $70 \%$ y el $90 \%$ sobre la situación de "pico", evitando el sobrecalentamiento del invernáculo. Al iniciar la fase de descarga, se observa que el sistema estabiliza la temperatura relativa $\mathrm{Tr}$ respecto de la situación base, sosteniendo el nivel térmico del ambiente durante toda la fase nocturna. Gráficamente, esto se advierte en la diferencia de $\operatorname{Tr}$ en ambos planteos, correspondiendo un incremento medio de entre el 25 y el $30 \%$ que se mantiene hasta el nuevo inicio del ciclo de trabajo. Así mismo, la temperatura relativa media del aire interior registró un incremento del $12 \%$ con la incorporación del sistema mixto.

\section{CONCLUSIONES}

El trabajo permitió el desarrollo de un sistema solar pasivo industrializable y de configuración versátil orientado a satisfacer requerimientos de climatización y/o estabilización térmica de espacios de uso residencial y productivo. Se pudieron diseñar y fabricar dos prototipos en función de los requerimientos planteados y el estudio del estado del arte en cuanto a tecnologías existentes. Los mismos, lograron mejorar los atributos de armado/montaje y modularidad; permitiendo mayor versatilidad de aplicación y diversidad de configuraciones respecto a la tecnología de referencia.

Se caracterizaron los sistemas y sus componentes estableciendo características termo-físicas y geométricas. Se llevó a cabo una transferencia al medio productivo a través de la interacción con una empresa de pre-moldeados de hormigón, en la que se desarrollaron los moldes y se fabricaron las piezas en hormigón vibrado $(\mathrm{H} 30)$ de manera industrializada para su posterior replicabilidad.

Se realizaron ensayos del sistema funcionando como muro acumulador de calor (aplicación al sector residencial) obteniendo resultados favorables en los niveles de retardo de la onda térmica ( $\sim \mathrm{hs}$.), rendimiento de carga del muro (60\%), y aporte térmico al ambiente interior. Se ensayó a su vez, la prestación del sistema funcionando como amortiguador térmico dentro de un invernadero liviano en laboratorio a cielo abierto. El análisis se realizó entre una Situación de Base, evaluando el invernadero sin ningún tipo de masa de acumulación de calor y con el sistema instalado, incorporando una masa térmica total de $864 \mathrm{~kg}$. El mismo demostró buena eficacia para el amortiguamiento térmico de este tipo de espacios productivos, estabilizando el ambiente en una temperatura relativa (interior/exterior) durante el período nocturno, entre un 25 y $30 \%$ superior a la situación en vacío, y evitando la inversión térmica de frecuente aparición en noches de cielo despejado. El aporte térmico medio neto durante el período nocturno se cuantificó en 36.22 MJ. Dicho aporte, evita el uso total o parcial de otros sistemas convencionales de climatización minimizando costos de producción. A su vez, la dinámica de carga/descarga del sistema permitió sostener el ambiente a partir del aporte térmico durante todo el período nocturno, amortigüando a 
su vez los picos diurnos en la temperatura del aire interior del invernadero. Durante el período diurno, con el sistema Mixto, fue registrada una estabilización de la temperatura interior con una disminución en el valor de "pico" que alcanzó un $90 \%$, en la variable de temperatura relativa interior/exterior. Este aspecto resulta de especial interés al permitir proteger los cultivos de daños por altas temperaturas sin necesidad de ventilar, al tiempo que se acumula dicho calor para ser liberado en la fase nocturna.

En síntesis, el trabajo demostró la viabilidad tecnológica del sistema propuesto, dando respuesta térmica y de climatización en los ensayos planteados en relación a su versatilidad de aplicación sobre espacios residenciales y agro-productivos. Se consideran alcanzados los objetivos del presente trabajo, obteniéndose un prototipo que demuestra empíricamente su utilidad para estas aplicaciones, y se prevé profundizar los estudios mediante ensayos y simulaciones del sistema en su aplicación como muro acumulador de calor (MAC) y en otras configuraciones dentro de espacios productivos.

\section{BIBLIOGRAFÍA}

Alanis, Saravia, \& Lesino. (1978). Material de alto calor específico para muro Trombe-Michel. Actas de 4ta. Reunión de trabajo de ASADES (págs. 241-248). La Plata: ASADES.

Balcomb, J. (1981). Chapter 16. Passive solar energy system for buildings. En Solar Energy Handbook. New York: ISBN 0-07-035474-X.

Bistoni, S., Iriarte, A., \& Saravia, L. (2004). Colector de aire con acumulador de agua para calefaccion de invernaderos. Avances en Energías Renovables y Medio Ambiente, 2.25-2.30.

Bourdeau, L., Jaffrin, A., \& Moisam, A. (1980). Capitage et stockage dénergie solaire dans I'habitat par le moyen de mur diode `a chaleur latente. Revue de Physique Appliquee, 15(3) 559-568.

Chévez, P. (2016). Análisis de medidas de eficiencia energética y energías renovables en el sector residencial. Arquisur. Concepción, Chile.

Discoli, C., \& Rosenfeld, E. (1986). Muro colector acumulador en agua: módulos industrializables de bajo costo. Actas $11^{a}$ Reunión de Trabajo de ASADES, (págs. 77-84). San Luis.

Discoli, C., \& San Juan, G. (2010). Patente $n^{\circ}$ IMPI 20110100010 - Muro climatizador Acumulador de Calor Mixto con vainas líquidas estancas. Argentina.

Discoli, C., Viegas, G., \& San Juan, G. (2012). Climatización por muros acumuladores de calor mixtos. IV Congresso Brasileiro de Energia Solar e V Conferencia Latino-Americana da ISES.

Discoli, Guerrero, Rosenfeld, \& Ravella. (1985). Muro colector acumulador en agua: su comportamiento en la zona templada-húmeda. Actas $10^{a}$ Reunión de Trabajo de ASADES, (págs. 15-22). Neuquen: ASADES.

Divecchia, P. R., \& Ruggi, D. (1979). Low and médium Temperature heat storage for solar application. Energia solare e nuove prospettive (pág. 473). Milano: Conferenza Internazionale de Milano.

Esteves, A., \& Gelardi, D. (1999). Muro de botellas de PET para incrementar la inercia termica en invernaderos para cultivos. Avances en Energías Renovables y Medio Ambiente, 9.3. 
Guerrero, Rosenfeld, Ravella, \& Cueto, D. (1979). Muro colector acumulador para el programa CESAD". . Actas 5ta. Reunión de trabajo de ASADES, (págs. 491-499). Córdoba, Argentina.

IPCC. (2014). Fifth Assessment Report (AR5). Intergovernmental Panel on Climate Change.

Juanicó, L., Piacentini, R., \& La Mattina, G. (2011). Desarrollo de invernaderos con sistema sustentable de confort térmico. Avances en Energías Renovables y Medio Ambiente, 3.01-3.08.

Lesino, Requena, Saravia, Fontanilla, \& Echazú. (1981). Edificios solares en la Puna: Estado de avance de su construcción y modelización. 7ma. Reunión de trabajo de Energía solar, ASADES, (págs. 138-153).

Li-Hong, G., Mei, Q., Hua-Zhong, R., Xiao-Lei, S., Qing-Yun , C., \& Zhen-Xian , Z. (2010). Structure, Function, Application, and Ecological Benefit of a Singl e-slope, Energy-efficient Solar Greenhouse in China. Hort Tecnologhy, June 2010 (20-3) 626-631.

Morse, E. (1881). Patente $n^{\circ}$ US Patent $N^{\circ} 246.626$ "Warming and ventilating apartemets by the sun's rays".Estados Unidos.

Suárez, H. (2012). Invernadero con acondicionamiento térmico solar. Avances en Energías Renovables y Medio Ambiente, 08.167-08.172.

Zalewski, L., Joulin, A., Lassue, S., Dutil, Y., \& Rousse, D. (2012). Experimental study of smallscale solar wall integrating phase change material. Solar Energy, 208-219. 